\title{
Fortalezas y dominios científicos, tecnológicos y humanísticos de la Universidad Técnica Estatal de Quevedo
}

\section{Strengths and Scientific, Technological and Humanistic Domains of Quevedo State Technical University}

\author{
*Washington Chiriboga Casanova ${ }^{1}$, Isabel Pérez Cruz ${ }^{1}$, Alina Martínez Hernández ${ }^{1}$ \\ ${ }^{1}$ Universidad Técnica Estatal de Quevedo. Facultad de Ciencias de la Ingeniería, Escuela Sistemas \\ Informáticos y Agroindustrial. Campus Ing. Manuel Haz Alvares, $k m 1.5$ vía Santo Domingo de Los \\ Tsáchilas. EC.120501. Quevedo, Ecuador \\ *Wchiriboga@uteq.edu.ec; iperez@uteq.edu.ec; amartinez @uteq.edu.ec
}

Rec.: 27.07.2016. Acept.: 25.10.2016.

Publicado el 30 de diciembre de 2016 Rev. Cienc. Soc. y Econ. UTEQ (2016), 1(1): 43-65

\section{Resumen}

La idea de identificar los dominios y fortalezas de la Universidad Técnica Estatal de Quevedo está relacionada con la intención de perfeccionar los procesos de gestión estratégica de la excelencia universitaria, cuya génesis se encuentra en los documentos fundacionales sobre la Educación Superior en el siglo XXI postulados por la UNESCO y asumidos como principios por los organismos rectores de la educación superior ecuatorianos, representativa, en su base epistemológica de la filosofía de la liberación latinoamericana y de la pedagogía social. El objetivo del trabajo se centra en identificar los dominios científicos, tecnológicos y humanísticos de la Universidad Técnica Estatal de Quevedo que servirán de fundamento para la planificación estratégica y prospectiva de los procesos sustantivos de la universidad. Desde una investigación descriptiva, de enfoque cualitativo y a partir de la conjunción de los métodos, se empleó el análisis de contenido y el cuestionario. Como resultado se identificaron los dominios científicos, tecnológicos y humanísticos de la UTEQ a través de su trayectoria académica e investigativa, su personal académico calificado, la infraestructura científica y la gestión pertinente del conocimiento. Los dominios científicos, tecnológicos y humanísticos de la UTEQ están en: el despliegue de la oferta académica en áreas, sectores estratégicos y de interés público; conocimientos con carácter interdisciplinario; evaluación y acreditación con categoría de B, equipamiento 
de laboratorios y servicios computacionales, modelo pedagógico por competencias, formación continua de los docentes, incremento de los programas de carrera y de maestría, impulso de las redes académicas y de investigación.

Palabras Clave: planificación estratégica, prospectiva, infraestructura científica, excelencia universitaria, gestión pertinente.

\begin{abstract}
The idea of identifying the domains and strengths of Quevedo State Technical University is related to the intention to perfect the processes of strategic management of university excellence, whose genesis can be traced back to the foundational documents on Higher Education in the XXI century postulated by UNESCO and assumed as principles by the governing bodies of Ecuadorian Higher Education, representative, in its epistemological basis, of Latin American liberation philosophy and social pedagogy, which integrate as a response to national and international challenges and tensions of the Contemporaneity. The research work aimed to identify the scientific, technological and humanistic domains of Quevedo State Technical University that will serve the foundation for strategic and prospective planning of substantive university processes. Based on descriptive research, with a qualitative approach which conjugates methods, according to the nature of this study, the content analysis and the questionnaire were used. As a result, the scientific, technological and humanistic domains of the UTEQ were identified, based on its academic and research trajectory, its qualified academic personnel, its scientific infrastructure and relevant knowledge management, all articulated with the substantive functions of the university. The scientific, technological and humanistic domains of the UTEQ are in: the academic programs in areas and strategic sectors of public interest; interdisciplinary knowledge; evaluation and accreditation with category of B; laboratory equipment and computer services; its pedagogical model based on competences, continuous teachers' staff training, increase of undergraduate and master's degree programs; impulse of academic and research networks.
\end{abstract}

Keywords: Strategic planning, prospective, scientific infrastructure, university excellence, relevant management. 


\section{Introducción}

La búsqueda de la excelencia, entendida esta como pleno acceso y democratización del conocimiento, calidad, pertinencia e innovación, responde la investigación para el perfeccionamiento de la actividad académica general que se lleva a cabo al interior de la propia universidad y que subyace a cada transformación, sea conceptual, metodológica o de gestión, ocurrida durante el proceso de consolidación del sistema de educación superior, en atención a las aspiraciones supremas de cualquier sociedad, con particular énfasis en la sociedad ecuatoriana (Daza, \& Arboleda, 2007).

Es por ello que el tratamiento del tema de los dominios y fortalezas universitarios prevé una obligada referencia a la evolución de la concepción sobre la excelencia de las instituciones universitarias (Alarcón \&, Luna, 2003) que se ha manejado desde el surgimiento de Consejo de Educación Superior y su trascendencia en los modelos y niveles de evaluación de la calidad y acreditación de instituciones y programas y que se ha expresado en la planificación estratégica de cada institución para el logro de estos indicadores (Knight, 2005).

El objetivo de este trabajo es identificar los dominios científicos, tecnológicos y humanísticos de la Universidad Técnica Estatal de Quevedo que servirán de fundamento para la planificación estratégica y prospectiva de los procesos sustantivos de la universidad relacionada con la formación profesional, posgraduada, la investigación científica, la innovación tecnológica y la vinculación con el entorno social. Así como la participación de la UTEQ como sujeto y actor de procesos de transformación social.

Es una investigación descriptiva, con enfoque cualitativo. Integra la utilización de métodos como el análisis y la síntesis de la bibliografía y documentos consultados, el método histórico - lógico, para la ubicación de la universidad desde su dimensión cultural e histórica, así como la descripción de las partes que conforman las fortalezas y sus características (Amaro \& García, 2013).

Se toma como guía metodológica para el proceso de elaboración de las fortalezas y dominios del UTEQ, la propuesta realizada por Larrea (2013), expuesta en el documento 
"Modelo de organización del conocimiento por dominios científicos, tecnológicos y humanísticos", la cual contiene las siguientes fases y actividades.

Las principales fuentes de información fueron los documentos institucionales como la Rendición de Cuentas 2015; el Informe final de la ejecución del plan de mejoras de la UTEQ 2016; el Informe de seguimiento a graduados, e informes de departamentos y de facultades sobre las fortalezas en cuanto a talento humano, líneas y proyectos de investigación e infraestructura que permite la actividad académica. Aplicando el método hermenéutico, se interpretaron los datos, para transformarlos en información comprensible (Arráez, 2006).

Se reseña en la literatura el trabajo de las universidades ecuatorianas en la consolidación de sus fortalezas y dominios como es el caso de la Universidad de Guayaquil, la Universidad Católica de Ecuador, la Universidad de San Francisco de Quito, la Universidad Politécnica Salesiana, la Universidad Técnica de Machala y la Universidad de Cuenca, por nombrar algunas (Consejo de Educación Superior, 2016).

El proceso de renovación de la Instituciones de Educación Superior ecuatorianas registra, como una de los problemáticas a resolver, la insuficiente pertinencia frente a los problemas de los entornos y contextos sociales, productivos y culturales, expresión de un trabajo fraccionado, aislado y asilado de los contextos históricos culturales (Camperos, 2012).

El Reglamento de Régimen Académico, del CES (Consejo de Educación Superior, Ecuador) genera una propuesta basada en la pertinencia, definida en el Art. 107 de la Ley Orgánica de Educación Superior (LOES, 2010), que establece:

El principio de pertinencia consiste en que la educación superior responda a las expectativas y necesidades de la sociedad, a la planificación nacional, y al régimen de desarrollo, a la prospectiva de desarrollo científico, humanístico y tecnológico mundial, y a la diversidad cultural. Para ello, las instituciones de educación superior articularán su oferta docente, de investigación y actividades de vinculación con la sociedad, a la demanda académica, a las necesidades de desarrollo local, regional y nacional, a la innovación y diversificación de profesiones y grados académicos, a las tendencias del mercado ocupacional local, regional y nacional, a las tendencias demográficas locales, provinciales 
y regionales; a la vinculación con la estructura productiva actual y potencial de la provincia y la región, y a las políticas nacionales de ciencia y tecnología (LOES, 2010, p. 19).

La UTEQ, asume la pertinencia, como un enfoque hacia las necesidades de la región, en concordancia con la planificación nacional y el régimen de desarrollo. De ahí, que las formulaciones para los rediseños de las carreras universitarias, está sustentada en la demanda académica, junto con las necesidades de desarrollo local, regional y nacional.

De la misma manera, el Reglamento de Régimen Académico en su Art. 78 en referencia a las fortalezas o dominios académicos, señala: Un dominio académico consiste en las fortalezas científicas, tecnológicas, humanísticas y artísticas demostradas por una IES, con base en su trayectoria académica e investigativa, personal académico altamente calificado, infraestructura científica y gestión pertinente del conocimiento. Las IES formularán su planificación institucional considerando los dominios académicos, los cuales podrán ser de carácter disciplinar e interdisciplinar (RRA-CES, 2015, p.32).

La dimensión social de la universidad, su proyección hacia el contexto y las comunidades se fundamenta en la integración de la ciencia, la tecnología, los saberes y los aprendizajes, como sustento nutricional de la esencia de la existencia de los centros e instituciones universitarios. El dinamismo de la integración se materializa en los llamados dominios académicos (Ramírez, \& Rivera, 2012).

La integración incorpora conocimientos, prácticas socioculturales y redes académicas como categorías que configuran los dominios científico-tecnológico-humanistas, y exigen nuevas configuraciones epistemológicas que deberán expresarse en la misión sustantiva de las IES (Institución de Educación Superior).

La dimensión espacio-contextual de los escenarios universitarios permite estructurar los dominios tomando en cuenta las fortalezas, capacidades y potencialidades de las IES, los contextos a los que responde el conocimiento, la organización de la ciencia y la tecnología, así como la integración a las redes de gestión académica y del saber.

Los investigadores asumen las matrices de abordaje metodológico para la determinación de los dominios que ofrece Larrea (2013), en ella los dominios estarán integradas por:

1. Áreas o sectores estratégicos 


\section{Conocimientos}

3. Trayectorias de capacidades o potencialidades

4. Las capacidades y potencialidades de la Universidad

5. Las redes- los tipos de redes académicas o de investigación a las que se vincula la Universidad.

6. La programación de los dominios científico, tecnológico y humanístico.

Los dominios se conciben como una red polisémica, compleja, histórica y cambiante de generación y gestión pertinente y notable del conocimiento científico, tecnológico y cultural, cuya estructura está centrada en redes académicas y científicas producidas desde, por y para las necesidades y dinámicas de los territorios locales, zonales, nacionales, regionales y mundiales (Guerrero, 2011).

\section{Materiales y métodos}

La investigación se desarrolla en el ámbito descriptivo de enfoque cualitativo y a partir de considerar en el orden teórico, el empleo de los métodos de analítico -sintético, inductivodeductivo, histórico-lógico, y la modelación, lo que evidencia la dimensión social de la universidad y su proyección hacia el contexto y las comunidades fundamentada en la integración de la ciencia, la tecnología, los saberes y los aprendizajes.

Dentro de los materiales (instrumentos), empleados se pueden señalar: la revisión de documentos, la observación, y el cuestionario, con el objetivo de identificar los dominios científicos, tecnológicos y humanísticos de la Universidad Técnica Estatal de Quevedo que servirán de fundamento para la planificación estratégica y prospectiva de los procesos sustantivos de la universidad.

\section{Resultados y discusión}

Como resultados de la aplicación de los métodos seleccionados para la investigación, se determinaron los siguientes dominios en la UTEQ: 


\section{Áreas o sectores estratégicos}

El diagnóstico de los núcleos potenciadores del buen vivir fortalece la investigación y organización del conocimiento de las IES y genera capacidades para organizar los clúster y las aplicaciones de las ciencias y tecnologías experimentales y de frontera de carácter inter y transdisciplinar, con plataformas y colectivos de inteligencia estratégica para la producción de innovación tecnológica y social (Caudillo, 2012).

Las tensiones y problemas expresados en los núcleos potenciadores del buen vivir, generan niveles de participación y de acción creativa de la ciudadanía para la transformación de la sociedad, con el uso extensivo e intensivo del conocimiento, las cuales pueden ser atendidas por los objetos de conocimiento de las carreras que oferta la UTEQ (Cuadro 1), (Plan Nacional del Buen Vivir, 2013- 2017).

Cuadro 1. Vinculación de la oferta académica con el Contexto y Objetivos del PNBV (Plan Nacional del Buen Vivir) y tensiones de la zona 5

\begin{tabular}{|c|c|c|c|}
\hline $\begin{array}{l}\text { Contextos } \\
\text { (Núcleos } \\
\text { potenciadores) }\end{array}$ & $\begin{array}{l}\text { Objetivos } \\
\text { PNBV }\end{array}$ & $\begin{array}{l}\text { Tensiones y } \\
\text { problemas } \\
\text { ZONA } 5\end{array}$ & Carrera \\
\hline & & $\begin{array}{l}\text { Desconcentración y } \\
\text { organización territorial }\end{array}$ & $\begin{array}{l}\text { Forestal Administración } \\
\text { Empresarial Economía, } \\
\text { Software, telemática }\end{array}$ \\
\hline & $\begin{array}{l}\text { Objetivo } 3: \text { Mejorar la } \\
\text { calidad de vida de la } \\
\text { población }\end{array}$ & $\begin{array}{l}\text { Medicina preventiva e } \\
\text { integral } \\
\text { Reducción de } \\
\text { enfermedades } \\
\text { infectocontagiosas }\end{array}$ & $\begin{array}{l}\text { Enfermería, Software, } \\
\text { Telemática }\end{array}$ \\
\hline \multirow[t]{3}{*}{$\begin{array}{l}\text { Derechos del } \\
\text { buen vivir y } \\
\text { hábitat } \\
\text { sustentable }\end{array}$} & \multirow{3}{*}{$\begin{array}{l}\text { Objetivo 7: Garantizar } \\
\text { los derechos de la } \\
\text { naturaleza y promover } \\
\text { la sostenibilidad } \\
\text { ambiental territorial }\end{array}$} & Banco de germoplasma & $\begin{array}{l}\text { Agronomía, } \\
\text { Agropecuaria, Zootecnia } \\
\text { Forestal }\end{array}$ \\
\hline & & $\begin{array}{l}\text { Tratamiento de aguas } \\
\text { servidas y residuos } \\
\text { sólidos } \\
\text { Gestión integral } \\
\text { desechos sólidos }\end{array}$ & $\begin{array}{l}\text { Ambiental, industrial, } \\
\text { mecánica, eléctrica, } \\
\text { Agroindustrias }\end{array}$ \\
\hline & & $\begin{array}{l}\text { Reforestación con } \\
\text { plantas endémicas }\end{array}$ & $\begin{array}{l}\text { Forestal, Agronomía, } \\
\text { Agropecuaria }\end{array}$ \\
\hline
\end{tabular}


Objetivo 10: Matriz productiva

\section{Economía social \\ y solidaria}

Objetivo 11: Asegurar la soberanía y eficiencia de los sectores estratégicos para la transformación industrial y tecnológica
Flujos y acuerdos comerciales nacionales inter-zonales para Administración, Contabilidad, Mercadotecnia, garantizar el acceso con Administración pública, , precios justos y estables Software, telemática a productos como café, maíz y trigo.

Fortalecimiento de pequeñas y medianas empresas en ramas estratégicas

Biotecnología para bioabonos, semillas certificadas, productos biodegradables y productos para bioremediación ambiental

Parque tecnológico para Alimentos, el procesamiento de Agroindustrial, productos alimenticios.

Industrial, Mecánica, Eléctrica, Seguridad industrial, Telemática, Software

Maricultura

Zootecnia, Agropecuaria

Producción de etanol y biodiesel

Agroindustrias, Industrial, Mecánica, Seguridad industrial

Producción orgánica de Agronomía, agropecuaria cultivos

Procesamiento de balsa Agroindustriales, y guayacán Mecánica, Industriales, Seguridad

Ecoturismo, turismo cultural, comunitario Turismo, Telemática, convencional, de playa, montaña y aventura

Uso potencial de energía renovable

Eléctrica, Mecánica, Software, Seguridad industrial, Telemática 


\section{Conocimientos}

La UTEQ, se plantea una organización del conocimiento de carácter inter y transdisciplinar, con el propósito de orientar los nuevos horizontes de la universidad, para el abordaje inmediato de las diferentes áreas formativas como, ingeniería, salud y administrativas, cuyas carreras están en el actual rediseño. Para el abordaje prospectivo se proyecta la creación de otras carreras. En el Cuadro (2) se expone la relación entre los campos de conocimiento y las carreras estratégicas y de interés público con que la universidad dará respuesta a las tensiones y problemas locales, regionales y nacionales.

Cuadro 2. Organización los dominios de conocimiento en función de los campos de conocimientos necesarios para atender los objetivos nacionales y regionales

\begin{tabular}{|c|c|c|c|}
\hline Campo amplio & Campo específico & Carrera & $\begin{array}{l}\text { Campos de } \\
\text { conocimiento }\end{array}$ \\
\hline $\begin{array}{l}\text { Tecnologías de la } \\
\text { información y } \\
\text { comunicación }\end{array}$ & TIC & $\begin{array}{l}\text { Software } \\
\text { Telemática }\end{array}$ & $\begin{array}{l}\text { Nuevas tecnologías de la } \\
\text { información y las } \\
\text { telecomunicaciones } \\
\text { ( } 2 \text { carreras) }\end{array}$ \\
\hline \multirow[t]{2}{*}{$\begin{array}{l}\text { Ingeniería, industria y } \\
\text { construcción }\end{array}$} & $\begin{array}{l}\text { Ingeniería y } \\
\text { profesiones afines }\end{array}$ & $\begin{array}{l}\text { Ambiental } \\
\text { Eléctrica } \\
\text { Mecánica }\end{array}$ & $\begin{array}{l}\text { Ciencias de la ingeniería } \\
\text { e industria ( } 8 \text { carreras) }\end{array}$ \\
\hline & $\begin{array}{l}\text { Industria y } \\
\text { producción }\end{array}$ & $\begin{array}{l}\text { Agroindustrias } \\
\text { Alimentos } \\
\text { Seguridad Industrial } \\
\text { Industrial }\end{array}$ & \\
\hline \multirow[t]{2}{*}{$\begin{array}{l}\text { Agricultura, } \\
\text { silvicultura, pesca y } \\
\text { veterinaria }\end{array}$} & Agricultura & $\begin{array}{l}\text { Agronomía } \\
\text { Agropecuaria } \\
\text { Zootecnia }\end{array}$ & $\begin{array}{l}\text { Ciencias agropecuaria y } \\
\text { forestal ( } 4 \text { carreras) }\end{array}$ \\
\hline & Silvicultura & Forestal & \\
\hline Salud y bienestar & Salud & Enfermería & Enfermería (1 carrera) \\
\hline Administración & $\begin{array}{l}\text { Educación } \\
\text { comercial y } \\
\text { administración }\end{array}$ & $\begin{array}{l}\text { Administración de } \\
\text { empresas } \\
\text { Contabilidad y auditoría } \\
\text { Mercadotecnia } \\
\text { Administración pública }\end{array}$ & $\begin{array}{l}\text { Ciencias sociales, } \\
\text { administrativas y } \\
\text { servicios ( } 6 \text { carreras })\end{array}$ \\
\hline Ciencias sociales & $\begin{array}{l}\text { Ciencias sociales } \\
\text { y del } \\
\text { comportamiento }\end{array}$ & Economía & \\
\hline Servicios & $\begin{array}{l}\text { Servicios } \\
\text { personales }\end{array}$ & Turismo & \\
\hline
\end{tabular}




\section{Trayectorias de capacidades o potencialidades}

Desde su creación, la UTEQ ha mejorado de manera sustantiva la calificación del recurso humano, ha aumentado y diversificado sus actividades académicas, ha potenciado sus programas de formación, y ha generado grupos consolidados en investigación, por lo que constituye un referente importante para el entorno en el que está insertada.

Lo planteado da cuenta de su ubicación en el grupo B, entre las universidades del país, luego del proceso de evaluación y acreditación del CEAACES (Consejo de Evaluación, acreditación y aseguramiento de la calidad de la Educación Superior. Ecuador) que se produce en virtud de sus indicadores académicos y su acreditación institucional por un período de cinco años, en las áreas de gestión, docencia de pregrado, investigación, docencia de postgrado y vinculación con el medio, cuya vigencia se extiende hasta el año 2018.

\section{Las capacidades y potencialidades de la Universidad, su infraestructura}

El desarrollo de la infraestructura en la UTEQ, busca armonizar las necesidades académicas y administrativas. La institución ha destinado anualmente importantes volúmenes de recursos hacia la inversión, como es el caso de infraestructura, cuyo propósito es ampliar y mejorar las instalaciones ofrecidas a la comunidad universitaria, incorporando los espacios necesarios para albergar nuevas carreras, aulas y laboratorios. Se efectuó la remodelación del campus "La María", el equipamiento avanzado en laboratorios y servicios computacionales existentes. Se realizaron adecuaciones en los laboratorios de Química, Bromatología, Física General, Biotecnología, Operaciones Unitarias, Robótica, Suelos y Aguas.

El sistema de bibliotecas cuenta con infraestructura de buena calidad, una apropiada dotación de recursos de información y disponibilidad de servicios de apoyo tecnológicos para incorporar en plenitud el uso de las TIC. Cuenta con una Biblioteca General con acceso a Internet, Con más de 13254 recursos bibliográficos y documentales que apoyan la formación académica y profesional. 


\section{Modelo Pedagógico por Competencias}

En la educación científica se exponen y experimentan los fundamentos de las ciencias, las metodologías y las aplicaciones para la investigación como sustento para el desarrollo de innovaciones y soluciones a los problemas que se deben enfrentar en el ejercicio de la profesión.

Los fundamentos teóricos que sustentan el Modelo Pedagógico por Competencias en la UTEQ consisten en la integración armónica de distintas teorías y enfoques pedagógicos, orientan, la elaboración y análisis de los programas de estudios; la sistematización del proceso de enseñanza-aprendizaje; y la comprensión de los programas de estudio. En esta sección se presentan los referentes filosóficos, psicopedagógicos y metodológicos que se tomaron en cuenta para el modelo pedagógico por competencias adoptado por la Universidad Técnica Estatal de Quevedo.

Desde la perspectiva de la UTEQ, el Modelo Pedagógico por Competencias es considerado: "como un proyecto abierto, integrador y flexible que ofrece una fundamentación integradora y dialéctica del proceso de enseñanza-aprendizaje, en coherencia con el contexto histórico-social y ajustado a las condiciones institucionales, a los avances del conocimiento científico, a la atención de las diferencias individuales, a la educación en valores en función de las aspiraciones de la sociedad contemporánea en torno a la formación integral de los estudiantes, desde las dimensiones académicas, prácticas e investigativas". (UPA-UTEQ, 2008).

Así, el currículo basado en competencias profesionales está orientado a la solución de problemas de manera integral, articulando los conocimientos generales, los conocimientos profesionales y las experiencias de trabajo desde una concepción humanista.

\section{Oferta académica}

La oferta académica de la UTEQ, ha incrementado el número de carreas y la población estudiantil (Cuadro 3). 
Cuadro 3. Carreras de grado y estudiantes de la UTEQ por facultad, periodos 2015-2016 (II semestre) y 2016-2017 (I semestre)

\begin{tabular}{|c|c|c|c|}
\hline Facultad & Carrera & $\begin{array}{l}\text { Pobl. } \\
\text { 2015-16 }\end{array}$ & $\begin{array}{l}\text { Pobl. } \\
\text { 2016-17 }\end{array}$ \\
\hline \multirow{5}{*}{ Ciencias Agrarias } & Agronomía (rediseño) & & 107 \\
\hline & Economía Agrícola (Vigente para titulación) & 104 & 97 \\
\hline & Ingeniería Agronómica & 429 & 388 \\
\hline & $\begin{array}{l}\text { Ingeniería en Administración de Empresas } \\
\text { Agropecuarias (Vigente para titulación) } \\
\text { Ingeniería en Horticultura y Fruticultura (Vigente } \\
\text { para titulación) }\end{array}$ & $\begin{array}{r}154 \\
1\end{array}$ & 137 \\
\hline & Total & 688 & 729 \\
\hline \multirow{4}{*}{$\begin{array}{l}\text { Ciencias } \\
\text { Ambientales }\end{array}$} & $\begin{array}{l}\text { Gestión Ambiental (Vigente para titulación) } \\
\text { Ingeniería en Ecoturismo (Vigente para } \\
\text { titulación) }\end{array}$ & 272 & 224 \\
\hline & Ingeniería Forestal & 260 & 308 \\
\hline & Licenciatura en Ecoturismo & 69 & 132 \\
\hline & Licenciatura en Gestión Ambiental & 76 & 138 \\
\hline \multirow{8}{*}{$\begin{array}{l}\text { Ciencias de la } \\
\text { Ingeniería }\end{array}$} & Total & 833 & 935 \\
\hline & $\begin{array}{l}\text { Ingeniería Agroindustrial } \\
\text { Ingeniería en Diseño Gráfico y Multimedia } \\
\text { (Vigente para titulación) } \\
\text { Ingeniería en Electricidad } \\
\text { Ingeniería en Seguridad Industrial y Salud }\end{array}$ & $\begin{array}{l}204 \\
201\end{array}$ & $\begin{array}{l}169 \\
204\end{array}$ \\
\hline & Ocupacional (Vigente para titulación) & 85 & 77 \\
\hline & Ingeniería en Sistemas & 189 & 188 \\
\hline & Ingeniería en Telemática & 118 & 100 \\
\hline & Ingeniería Industrial & 261 & 262 \\
\hline & Ingeniería Mecánica & 166 & 169 \\
\hline & Total & 1424 & 1397 \\
\hline \multirow{9}{*}{$\begin{array}{l}\text { Ciencias } \\
\text { Empresariales }\end{array}$} & Economía & 328 & 375 \\
\hline & $\begin{array}{l}\text { Gestión Empresarial (Vigente para titulación) } \\
\text { Ingeniería en Administración Financiera (Vigente }\end{array}$ & 555 & 523 \\
\hline & para titulación) & 197 & 138 \\
\hline & $\begin{array}{l}\text { Ingeniería en Contabilidad y Auditoría - CPA } \\
\text { (Vigente para titulación) }\end{array}$ & 499 & 477 \\
\hline & Licenciatura en Contabilidad y Auditoría - CPA & 76 & 164 \\
\hline & Licenciatura en Gestión Empresarial & 82 & 167 \\
\hline & Licenciatura en Marketing & & 41 \\
\hline & Marketing & 133 & 121 \\
\hline & Total & 1870 & 2006 \\
\hline \multirow{6}{*}{ Ciencias Pecuarias } & Ingeniería Agropecuaria (rediseño) & & 92 \\
\hline & Ingeniería Agropecuaria & 316 & 268 \\
\hline & Ingeniería en Alimentos & 258 & 314 \\
\hline & Ingeniería Zootécnica & 215 & 176 \\
\hline & Ingeniería Zootécnica (rediseño) & & 69 \\
\hline & Total & 789 & 919 \\
\hline \multirow{19}{*}{$\begin{array}{l}\text { Unidad de Estudios } \\
\text { a Distancia }\end{array}$} & $\begin{array}{l}\text { Administración y Gestión Pública y Municipal } \\
\text { (Vigente para titulación) }\end{array}$ & 38 & 5 \\
\hline & Gestión Empresarial & 348 & 310 \\
\hline & $\begin{array}{l}\text { Ingeniería Agropecuaria (Semipresencial) } \\
\text { (Vigente para titulación) }\end{array}$ & 392 & 347 \\
\hline & Ingeniería Comercial (Vigente para titulación) & 79 & 2 \\
\hline & Ingeniería en Administración de Recursos & & \\
\hline & Humanos (Vigente para titulación) & 50 & \\
\hline & Ingeniería en Administración Turística y & & \\
\hline & Empresas de Recreación (Vigente para titulación) & 17 & 1 \\
\hline & $\begin{array}{l}\text { Ingeniería en Contabilidad y Auditoría - CPA } \\
\text { (Vigente para titulación) }\end{array}$ & 417 & 362 \\
\hline & Ingeniería en Sistemas (Vigente para titulación) & 219 & 188 \\
\hline & Ingeniería Industrial (Vigente para titulación) & 156 & 122 \\
\hline & $\begin{array}{l}\text { Licenciatura en Contabilidad y Auditoría - CPA } \\
\text { Licenciatura en Enfermería (Vigente para }\end{array}$ & 94 & 147 \\
\hline & titulación) & 66 & 5 \\
\hline & Licenciatura en Gestión Empresarial & 101 & 155 \\
\hline & Licenciatura en Marketing & 30 & 74 \\
\hline & Marketing & 247 & 200 \\
\hline & $\begin{array}{l}\text { Secretariado Ejecutivo en Técnicas Informáticas } \\
\text { (Vigente para titulación) }\end{array}$ & 30 & \\
\hline & Total & 2290 & 1918 \\
\hline & & 7894 & 7905 \\
\hline
\end{tabular}

Fuente: Sistema Control Académico Universitario - SICAU.

Nota: Corte de datos al 13 de junio del 2016 
La distribución de las carreras por área de conocimiento (Figura 1) destaca el elevado peso de las ciencias agropecuaria y forestal en la oferta académica.

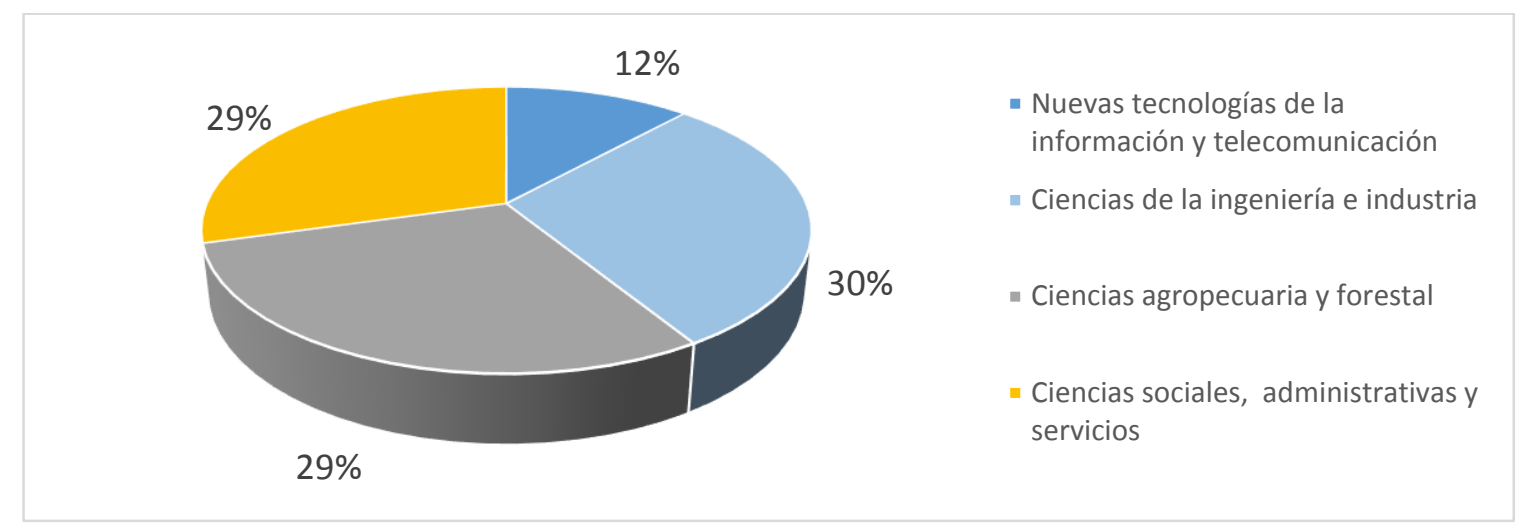

Figura 1. Proporción de carreras por área de conocimiento

Fuente: Síntesis documental

En el Cuadro (4) se detalla la formación de posgrado, donde aparecen los programas de maestría aprobados para impartir en la UTEQ.

\section{Cuadro 4. Programas de posgrado (maestría)}

\begin{tabular}{|c|c|}
\hline Maestría & Resolución de aprobación \\
\hline 1. Maestría en Desarrollo del Medio Ambiente & $\begin{array}{l}\text { Resolución Décima Segunda del OCAS, del } 3 \text { de } \\
\text { junio del } 2014\end{array}$ \\
\hline 2. Maestría en Contabilidad y Auditoría & $\begin{array}{l}\text { Resolución Vigésima Quinta del OCAS, del } 16 \text { de } \\
\text { septiembre del } 2014 . \\
\text { Resolución CES RPC-SO-03-No. 033-2016, del } \\
20 \text { de enero del } 2016 .\end{array}$ \\
\hline $\begin{array}{l}\text { 3. Maestría en Gerencia de Innovaciones } \\
\text { Educativas }\end{array}$ & $\begin{array}{l}\text { Resolución Vigésima Sexta del OCAS, del } 16 \text { de } \\
\text { septiembre del } 2014\end{array}$ \\
\hline $\begin{array}{l}\text { 4. Maestría en Silvicultura, Mención: Manejo y } \\
\text { Aprovechamiento Forestal. }\end{array}$ & $\begin{array}{l}\text { Resolución Centésima del OCAS, del } 6 \text { de enero } \\
\text { del } 2015\end{array}$ \\
\hline $\begin{array}{l}\text { 5. Maestría en Desarrollo de Aplicaciones con } \\
\text { Software Libre }\end{array}$ & $\begin{array}{l}\text { Resolución Décima Tercera del OCAS, del } 1 \text { de } \\
\text { septiembre del } 2015 .\end{array}$ \\
\hline
\end{tabular}

Fuente: Síntesis documental

\section{Formación continua de los docentes}

Durante este periodo, la universidad diseñó de manera participativa, un Sistema de Evaluación Integral del desempeño Académico, sobre la base de los lineamientos establecidos en el Reglamento de Escalafón Docente expedido por el CES. 
La UTEQ elaboró el Reglamento de Evaluación Integral de Desempeño Docente, aprobado por el Consejo Universitario, con Resolución cuadragésima tercera del 19 de agosto de 2014. El mismo permite valorar la producción docente, científica, la vinculación, la gestión administrativa y académica.

Un total de 64 docentes fueron recategorizados en la Universidad, entre los años 2014 y 2015, lo que en relación al número total de profesores titulares constituye el 34,22\%. Las remuneraciones mensuales promedio de un buen número de los profesores e investigadores a tiempo completo de la UTEQ han sido notablemente incrementadas, en relación a la situación inicial previa al período de ejecución del plan de mejoras, lo que ha permitido cumplir el indicador Remuneración, tanto del Modelo de Evaluación Institucional CEAACES proceso 2013, como del Modelo de recategorización.

La formación de doctores se observa en la Figura 2 y refleja la intención de la institución por potenciar la formación de doctores en los proyectos vinculados con los cambios de la matriz productiva del país.

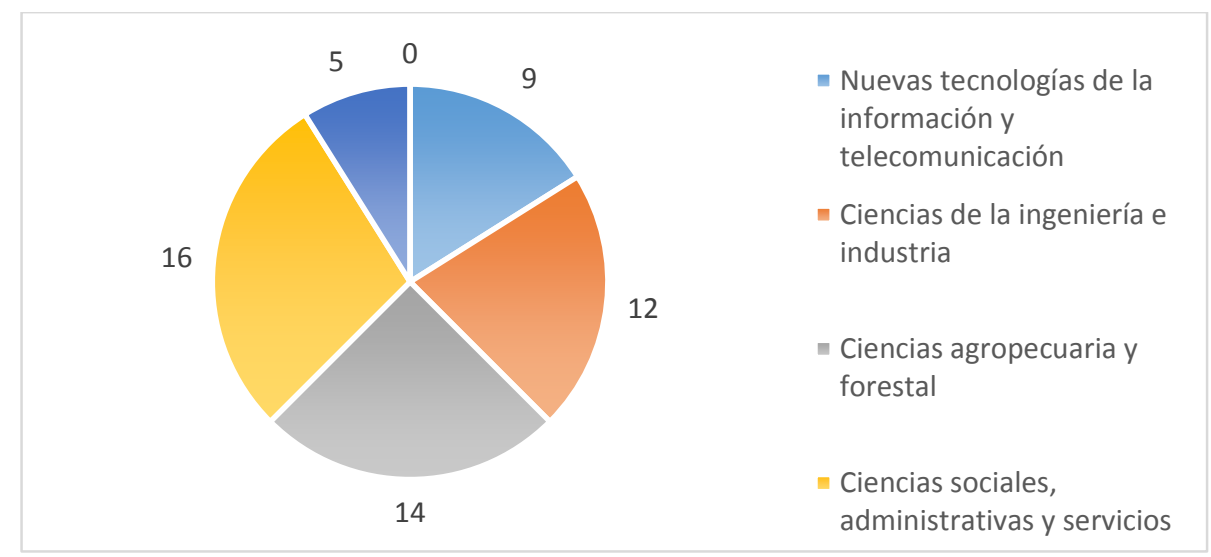

Figura 2. Proporción de la formación de docentes cursando Doctorados relacionados con áreas de Conocimiento. Fuente: Síntesis documental

\section{Las Redes académicas, tipos de redes académicas o de investigación a las que se vinculan la Universidad}

La UTEQ ha venido participando activamente en las redes académicas conformadas por las universidades ecuatorianas con acompañamiento de la SENESCYT para la elaboración de los proyectos de diseño o rediseño de la oferta académica, su armonización a partir de las demandas del entorno y de los procesos de acreditación relacionada con los Indicadores 
Institucionalización y Gestión de Recursos para la Investigación, constantes en los Modelos de Evaluación Institucional CEAACES, tanto del proceso 2013 como de la Recategorización 2015.

La institución participa en la Red Ecuatoriana de Carreras de Sistemas, Informática y Computación (RECSIC); Red Académica de Carreras de Ingeniería Eléctrica del Ecuador (RACIEE); Red Ecuatoriana de Carrera de Ingeniería Electrónica, Telecomunicaciones y Afines (RECIETA); Red Académica Ecuatoriana de las carreras del Campo de Administración (RAECA) ; Red Académica Nacional de Idiomas (RANI); Red Académica Ecuatoriana de Carreras de Ingeniería Forestal (RACIFOR); Red Académica de carreras de Ingeniería Ambiental (REDCCAR); Académica Ecuatoriana de carreras en Turismo y Hospitalidad (RAECTH); Red de Carreras de Ingeniería Agropecuaria (REDCIAPE); Red de Carreras de Ingeniería Zootécnica (RED ZOOTECNIA); Red de Carreras de Ingeniería de Ingeniería de Alimentos (RED CIALI); Red Carrera Ingeniería Agronómica del Ecuador y la Red Académica Ecuatoriana de Economía (RAEDE).

\section{Redes de investigación}

Se ha impulsado la participación proactiva de la UTEQ en seis redes de investigación: Red de Universidades para la Investigación y Posgrado (RUIP); Red Interinstitucional de Biotecnología (SENESCYT); Red Interinstitucional Agropecuaria, Agroindustria y Pesca (SENESCYT); Red de Metalmecánica y Astilleros (SENESCYT); Asociación ecuatoriana de Producción Animal (AEPA); Red sobre la Conservación de la Biodiversidad de los Animales Domésticos y Locales para el Desarrollo Rural Sostenible (CONBIAND Iberoamérica).

La participación en redes ha contribuido a los logros cualitativos y cuantitativos en investigación, la consolidación de la actividad investigativa en ciencias aplicadas, campos en que la UTEQ tiene fortalezas y que constituyen los dominios de la institución (agronomía, forestal, zootecnia, alimentos, biotecnología) el incremento de proyectos desarrollados mediante fondos concursables, el aumento de publicaciones y la consolidación de las estructuras de apoyo e instrumentos para la investigación, innovación y emprendimiento. 
Por otra parte, la UTEQ mantiene alianzas estratégicas para la organización de eventos científicos de difusión de avances en distintas áreas, así tenemos: La Universidad de Granada (España), Universidad de La Coruña (España), Universidad Autónoma de Guerrero (México) y la Universidad de la Habana (Cuba), con las cuales la UTEQ organizó el Taller Iberoamericano de Investigación de Operaciones. (Noviembre, 2015) celebrado en Quevedo.

\section{Líneas de Investigación}

La UTEQ tiene actualmente catorce líneas de investigación, ordenadas por áreas, de la siguiente forma:

Líneas de investigación agrícola: Desarrollo y manejo de variedades e híbridos en cultivos de interés estratégico para el Ecuador; desarrollo de conocimiento y tecnologías de agricultura alternativa aplicable a las condiciones del trópico húmedo y semihúmedo del litoral ecuatoriano; desarrollo de sistemas de producción que promuevan el uso eficiente de los recursos genéticos y ambientales (suelo, agua, luz).

Líneas de investigación educativa: Investigación educativa sobre planificación, ejecución y evaluación curricular; aseguramiento de la calidad educativa en las funciones universitarias.

Líneas de investigación empresarial: Estudio de los factores que impulsan el desarrollo económico y social de los grupos poblacionales del área de influencia de la UTEQ; emprendimiento y administración estratégica de empresas.

Líneas de investigación ambiental: Evaluación de la calidad del suelo, agua y aire y alternativas de mitigación y estudio; mejora genética y silvicultura de especies forestales y florísticas nativas, y exóticas, en el trópico húmedo ecuatoriano.

Líneas de investigación pecuaria: Desarrollo de acciones de conservación, uso y mejora productiva de los recursos zoogenéticos criollos de las especies domésticas de interés del trópico ecuatoriano; comportamiento agronómico, evaluación y mejoramiento de las características nutricionales y métodos de conservación de gramíneas, leguminosas, árboles forrajeros, subproductos agropecuarios y residuos agroindustriales con fines de 
alimentación de los animales domésticos; evaluación y mejoramiento de las limitantes de la salud y productividad de las diferentes especies animales en los sistemas de producción del trópico y subtrópico ecuatoriano y su relación con la salud humana.

Líneas de investigación en las ciencias de la ingeniería: Desarrollo de tecnología para la transformación de la materia prima agroindustrial; ambientes informáticos para aplicaciones multimedia e inteligencia artificial.

\section{Producción Científica}

La producción científica de un país o institución es el conjunto de sus trabajos publicados, en tanto resultados de un proceso de investigación, y los indicadores bibliométricos las medidas que proveen información sobre esos resultados (Spinak, 1996). Una de las principales ventajas de estos indicadores es que permiten observar, describir, evaluar y monitorear el estado y evolución de dominios científicos en diferentes niveles de agregación geográficos, institucionales o temáticos. Asimismo, y a través de la comparación, estos indicadores pueden dar cuenta de la evolución y posicionamiento científico de un país con respecto al mundo, de una institución en relación con su país.

El Cuadro (5) ilustra los resultados en artículos publicados en los últimos años.

\section{Cuadro 5. Publicación de artículos}

\begin{tabular}{lcccc}
\hline Artículos & $\mathbf{2 0 1 3}$ & $\mathbf{2 0 1 4}$ & $\mathbf{2 0 1 5}$ & $\mathbf{2 0 1 6}$ \\
\hline Latindex & 9 & 20 & 33 & 20 \\
Scopus & 10 & 8 & 11 & 7 \\
TOTAL & 19 & 28 & 44 & 27 \\
\hline
\end{tabular}

Fuente: Síntesis documental

En cuanto a la concentración de la producción científica por áreas de conocimiento, tomando como referencia el número total de artículos publicados en el período 2015-2016 en revistas con índice de alto impacto, el $40 \%$ corresponde a Agricultura, el $25 \%$ a Zootecnia, el 15\% a Forestal, el 10\% a Alimentos, y el 10\% al área de Informática. 


\section{Proyectos de Investigación}

La UTEQ ha realizado cuatro convocatorias de proyectos de investigación en el transcurso de los últimos años, en total se aprobaron once proyectos de investigación. Se tabularon los datos para ver los resultados de proyectos por áreas de conocimiento (Figura 3).

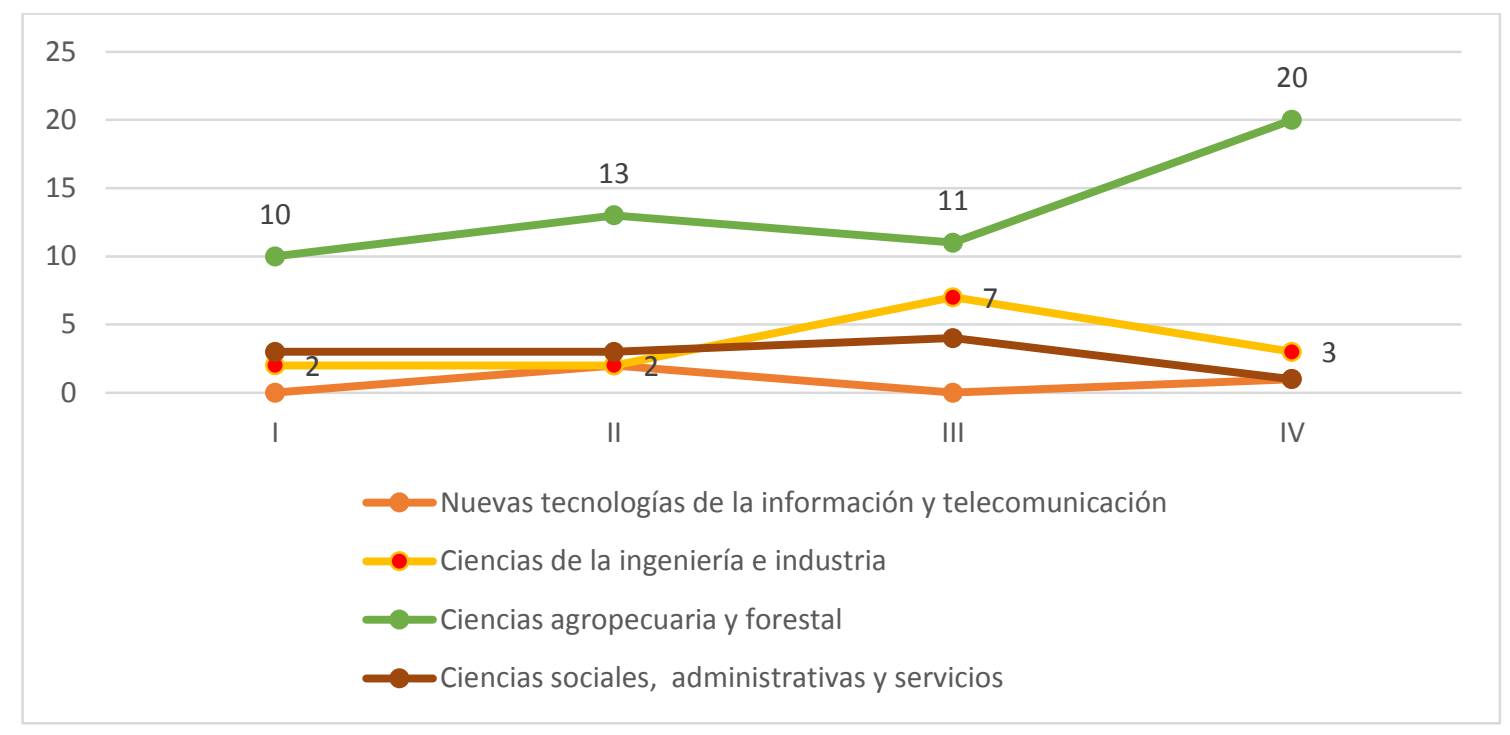

Figura 3. Proyectos de investigación por Áreas de conocimiento

Fuente: Síntesis documental

\section{Subsistema de vinculación social}

La interrelación de docencia, investigación y vinculación la alcanza la UTEQ, a través de los convenios y proyectos de vinculación. Esto significa una visión sistémica de la formación profesional, mediante la investigación científica y la reflexión crítica sobre la propia práctica profesional. Los proyectos de vinculación están relacionados con áreas del conocimiento como: nuevas tecnologías de la información y telecomunicación; ciencias de la ingeniería e industria; ciencias agropecuaria y forestal; ciencias sociales, administrativas y servicios de salud. Abarcan un total de veinte dos proyectos de vinculación.

\section{Inserción laboral - seguimiento a graduados}

La UTEQ cuenta con un sistema de seguimiento a graduados que permite medir las competencias de empleabilidad, la trayectoria laboral pertinente, la calidad y necesidades de perfeccionamiento del graduado $\mathrm{y}$, de esta forma, retroalimentar permanentemente el currículo y la oferta de pre y postgrado. 
Del total de graduados en la UTEQ, un 63\% se encuentra laborando (esto debido a que un alto porcentaje recién se ha graduado en el año 2015) y con respecto a la naturaleza de las empresas, en las cuales se encuentran ejerciendo su profesión, tenemos que un $62 \%$ trabajan en empresas privadas, un $29 \%$ en empresas públicas y un $9 \%$ en empresas propias. El grado de satisfacción de los graduados con su formación profesional es de un 90\%. Los informes de seguimiento de graduados han sido utilizados para el rediseño curricular de las carreras, lo que ha contribuido al cumplimiento del indicador de oferta académica, de acuerdo al modelo de evaluación del CEAACES, tanto del proceso de evaluación del año 2013, como del modelo de recategorización del 2015.

Asimismo, del total de graduados, el $75 \%$ se encuentra trabajando en empresas relacionadas con la formación recibida en la UTEQ. El 46\% de los graduados tienen más de tres años laborando en sus empresas, lo que indica una relativa estabilidad en la empresa. El $8 \%$ tiene tres años, el 15\% dos años, el 13\% 1 año, y el 18\% tiene solo meses en el mercado de trabajo.

Las carreras que más se destacan por el número de graduados y el porcentaje de empleabilidad son: Ingeniería Agropecuaria con el 85.51\%, Ingeniería en Sistemas con $83.33 \%$ e Ingeniería Industrial con $89.47 \%$.

La Unidad de Estudios a Distancia posee un alto porcentaje de empleabilidad en las carreras que oferta. En esto sobresale la carrera de Contabilidad y Auditoría que cuenta con un total de 86 graduados en el ámbito laboral, equivalente al $85.15 \%$.

Adicional al estudio de empleabilidad, es necesario identificar si la formación recibida está vinculada con el trabajo que desempeña (Figura 4). 


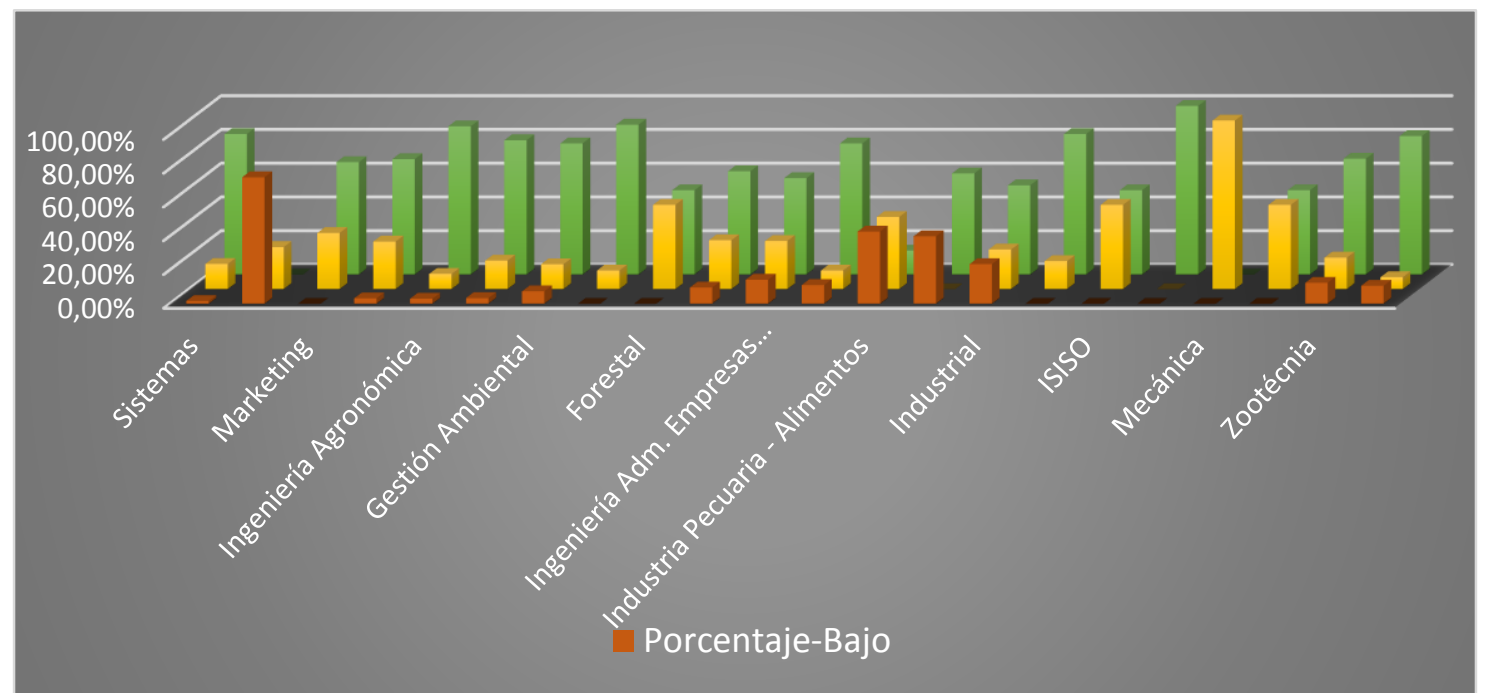

Figura 4. Porcentaje de relación del trabajo con la formación académica de los graduados de la UTEQ, clasificados por carreras. GRADUTEQ 2015

Fuente: Síntesis documental

\section{La Programación de los dominios científico, tecnológico y humanístico}

Tomando en cuenta las potencialidades de la UTEQ, así como las demandas de desarrollo de la zona 5 y las áreas de influencia de la universidad, referidas en el PNBV, Agenda Zonal y planes regionales, se programan los siguientes dominios: la Producción desde la transformación de la matriz productiva y la soberanía alimentaria; la Industrialización con la mecanización, diseño y construcción de maquinarias y la gestión de los agentes contaminantes de la industria; la Superación de la brecha tecnológica para asegurar la soberanía y eficiencia de los sectores estratégicos; la Competitividad a través de una mejor planificación territorial; la Preservación de los recursos naturales y el Vivir Saludable mediante gestión de salud preventiva y de atención básica.

\section{Dominio en el desarrollo regional}

La relación de la UTEQ con los proyectos de desarrollo regional está dada en la participación potencial en los proyectos estratégicos nacionales. En la figura 5a se presentan las potencialidades de los proyectos regionales y en la figura $5 \mathrm{~b}$ con los proyectos territoriales. 


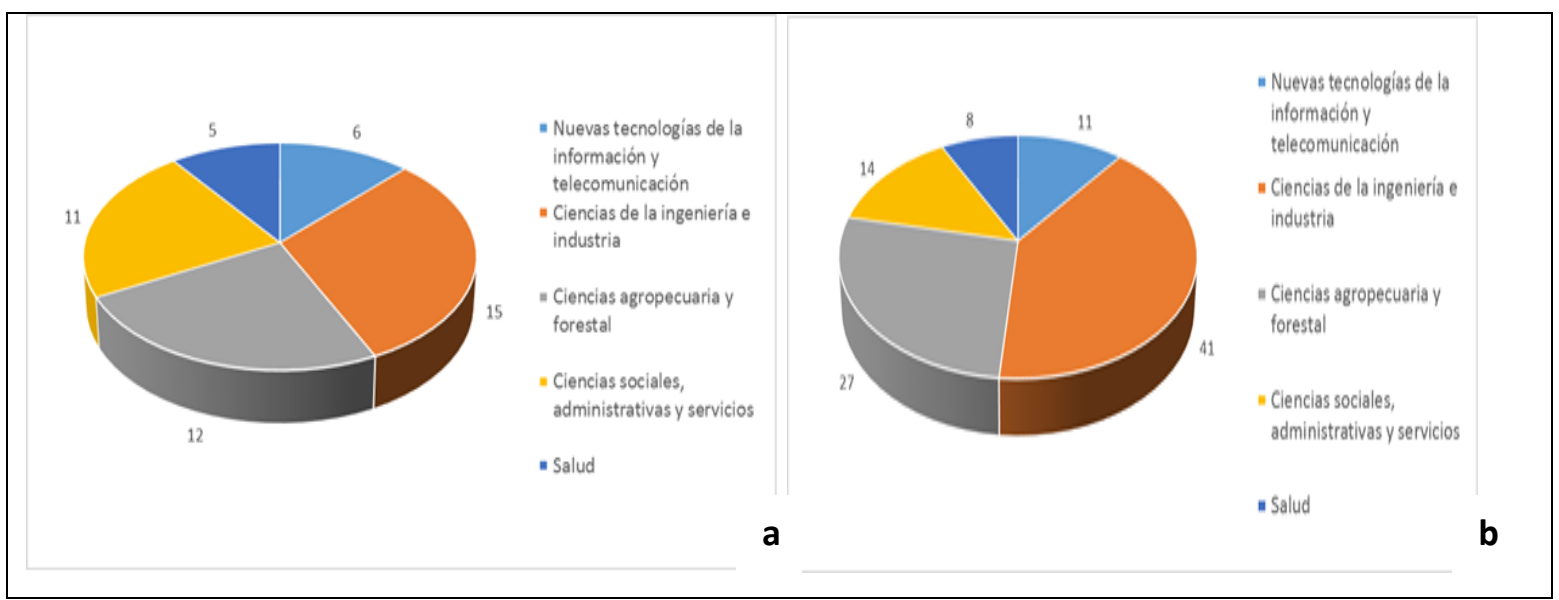

Figura 5. Proyectos Regionales (a), Proyectos territoriales, Zona 5 (b)

Fuente: Síntesis documental

\section{Conclusiones}

Los dominios científicos, tecnológicos y humanísticos sirven de fundamento para la planificación estratégica y prospectiva de los procesos sustantivos de la universidad Técnica Estatal de Quevedo.

La programación de los dominios científico, tecnológico y humanístico está relacionada con la formación profesional, posgraduada, la investigación científica, la innovación tecnológica y la vinculación con el entorno social.

El desarrollo de un Modelo pedagógico por competencias fortalece la formación humanista, desde el cual se ofrecen 46 programas de carreras y 5 programas de maestrías. En la formación continua del claustro de los docentes el 43, $22 \%$ se ha recategorizado y 51 docentes cursan estudios de doctorados.

La participación en redes de investigación consolida la actividad investigativa, junto a de 13 redes académicas, 14 líneas de investigación, 11 proyectos de investigación y 22 proyectos de vinculación, trabajo científico que se refleja en el incremento del número de artículos publicados en revistas de prestigio internacional.

La programación de los dominios está orientada hacia aspectos del desarrollo como la producción, la industrialización, la superación de la brecha tecnológica, la competitividad, la prevención de los recursos naturales y el buen vivir saludable, y la incorporación de la universidad a través de la participación en proyectos de desarrollo regional y territorial. 


\section{Referencias bibliográficas}

Alarcón, A. \&, Luna, J. (2003). Antecedentes, Situación Actual y perspectivas de la evaluación y acreditación de la educación superior en Centroamérica, Secretaría Permanente del CSUCA, Guatemala.

Amaro, R, \&, García, C. (2003). Competencias del docente universitario para enseñanza en entornos virtuales en el aprendizaje en el contexto universitario. Málaga, Ediciones Aljibe. 181 pp.

Arráez, M. (2006). La Hermenéutica, una actividad interpretativa. Revista Universitaria de Investigación, 7(2): 171-181, Universidad Pedagógica Experimental libertador, Caracas, Venezuela.

Asamblea Nacional del Ecuador. (2010). Ley Orgánica de Educación Superior. Quito: Registro Oficial No 298. P 19

Campero, M. (2012). El proyecto de evaluación e investigación evaluativa, sus componentes básicos. Editorial. Chacín Caracas, Venezuela.

Caudillo, F. \& Gloria, A. (2012). El buen vivir: un diálogo intercultural Ra Ximhai, 8(2): 345-364, Buenos Aires, CLACSO.

Comisión General de Evaluación Interna. (2016). Informe Final de la ejecución del plan de Mejoras de la UTEQ. Quevedo: UTEQ.

Consejo de Educación Superior. (2016). Reglamento de Régimen Académico. Ecuador. Quito: CES.

Cuchumbè, N. J. (2007). La dimensión interpretativa como horizonte epistemológico: el reconocimiento de la diferencia. Praxis filosòfica, no.25 Cali July/Dec. 2007.

Daza, S., Arboleda, T. (2007). Comunicación pública de la ciencia y la tecnología en Colombia: ¿políticas para la democratización del conocimiento? Signo y Pensamiento, 26(50): 100-125. Pontificia Universidad Javeriana, Bogotá, Colombia.

De Miguel, M. (2005, p. 19). Modalidades de enseñanzacentradas en el desarrollo de competencias. Oviedo: Ubniversidad de Oviedo.

García, J. (2014). "El Paradigma Humanista en la Educación. Universidad de Guanajuato. Revista Liceus. Madrid.

Jaramillo, L. (2008, p. 12). ¿Qué es Epistemología?. Mi mirar epistemológico y el progreso de la ciencia. Obtenido de Cinta de Moebio: www.philpapers.org/rec/GUIQE 
Larrea de Granados, E. (2013, p. 2). Modelo de organización del conocimiento por dominios científicos, tecnológicos y humanísticos. Quito: Consejo de Educación Superior.

Larrea, E. (2013). Modelo de Organización del Conocimiento por Dominios Científicos, Tecnológicos y Humanísticos. Obtenido de CES: www.ces.gob.ec/doc/ Noviembre/conocimiento\%20por\%20dominios\%20cientficos.pdf

Larrea, E. (2015). Propuesta del currículo genérico para las carreras de educación. Quito: Consejo de Educación Superior.

Marrero, E. (2014). ampliaciòn de horizontes epistemològicos para la construcciòn de nuevos entornos organizativos en instituciones educativas universitarias . Panorama, 8 (14): 59-72.

Plan nacional para el buen vivir 2013-2017, TOMO I .Resolución 2, Registro Oficial Suplemento 78 de 11-sep 2013 Estado: Vigente

Realpe, SI. (2011). Evolución conceptual de los paradigmas de las. Bucaramanga: Universidad Industrial de Santander.

Registro, O. (2010,p. 19). Ley Orgánica de Educación Superior. Quito: Registro Oficial.

RRA-CES. (2015). Reglamento de Régimen Académico. Quito: Consejo de Educación Superior.

Serna, M. (2003). El Modelo por competencias en el ITSON. Perspectiva Universitaria, ITSON.

Universidad Técnica Estatal de Quevedo. (2015). Estatuto de la Universidad Técnica Estatal de Quevedo. Quevedo: UTEQ.

UPA - UTEQ. (2012). Plan Estratégico de Desarrollo Institucional. Quevedo: Unidad de Planificación y Evaluación de la Universidad Técnica Estatál de Quevedo.

UPA-UTEQ. (2008). Modelo Pedagógico por Competencias de la Universidad Técnica Estatal de Quevedo. Quevedo: UTEQ. 